\title{
Sodium-glucose Co-transporter-2 Inhibitors in Type 1 Diabetes-a Dangerous Ally
}

Gagan Priya, ${ }^{1}$ Sanjay Kalra, ${ }^{2}$ and Vishal Bhambri ${ }^{1}$

1. Fortis Hospital, Mohali, India; 2. Bharti Hospital, Karnal, India

DOI: https://doi.org/10.17925/USE.2017.13.02.75

$\mathrm{T}$ here is an unmet need for adjunctive non-insulin-based therapies in type 1 diabetes (T1D). Weight gain, recurrent hypoglycemia and suboptimal glycemic control remain significant challenges. Sodium-glucose co-transporter-2 (SGLT2) inhibitors and dual inhibitors of sodium-glucose co-transporter-1 (SGLT1) and SGLT2 may have a potential role as an add-on therapy to insulin. The benefits include improved glycemic control, weight reduction, and reduced insulin dose requirement. However, the risk of diabetic ketoacidosis with SGLT2 inhibitors is significant and the diagnosis may be delayed due to absence of significant hyperglycemia. At present, SGLT2 inhibitors are not approved for use in T1D, and the risks should be discussed at length with the patient. We propose strategies to minimize the risk of diabetic ketoacidosis associated with off-label use of SGLT2 inhibitors in T1D.

\section{Keywords}

Type 1 diabetes, diabetic ketoacidosis, euglycemic diabetic ketoacidosis, sodium-glucose co-transporter-2 (SGLT2) inhibitors

Disclosure: Gagan Priya, Sanjay Kalra, and Vishal Bhambri have nothing to declare in relation to this article. No funding was received in the publication of this article.

Compliance with Ethics: This study involves a review of the literature and did not involve any studies with human or animal subjects performed by any of the authors.

Authorship: All named authors meet the International Committee of Medical Journal Editors (ICMJE) criteria for authorship of this manuscript, take responsibility for the integrity of the work as a whole, and have given final approval to the version to be published.

Open Access: This article is published under the Creative Commons Attribution Noncommercial License, which permits any noncommercial use, distribution, adaptation, and reproduction provided the original author(s) and source are given appropriate credit.

Received: September 4, 2017

Accepted: November 13, 2017

Citation: US Endocrinology, 2017;13(2):75-8

Corresponding Author: Gagan Priya, Fortis Hospital, Mohali, India. E: gpriya77@gmail.com
There is a rising trend of overweight and obesity in individuals with type 1 diabetes (T1D). The prevalence of overweight and obesity among newly diagnosed T1D subjects was 21-22\% in the 2-19 year age group in the Pediatric Diabetes Consortium and the SEARCH for Diabetes in Youth study. ${ }^{1,2}$ This has been attributed to a general worldwide increase in prevalence of obesity and similar risk factors such as family history, ethnicity, and sedentary lifestyle may be contributory. But several other factors related to insulin treatment may further exacerbate weight gain. Hypoglycemia and defensive snacking result in a state of calorie excess. Over-insulinization to achieve glycemic targets compounds the problem. In the DCCT (NCT00360815) and EDIC (NCT00360893) trials, excessive weight gain was associated with an increase in central obesity, insulin resistance, dyslipidemia, hypertension, and atherosclerosis. ${ }^{3}$ Approximately $50 \%$ patients with T1D have features of metabolic syndrome. ${ }^{4}$

\section{Is there a need for adjunctive therapy in type 1 diabetes?}

T1D is caused by autoimmune destruction of pancreatic $\beta$-cells and consequent absolute insulin deficiency. Insulin, therefore, remains the cornerstone of treatment. However, despite significant advances in the availability of more physiological insulin preparations, insulin delivery devices including continuous subcutaneous insulin infusion (CSII) pumps and continuous glucose monitoring (CGM) systems, most patients fail to achieve glycemic targets. ${ }^{5}$ There are periods of hyperglycemia and hypoglycemia with marked daily fluctuations in blood glucose. Weight gain associated with insulin, severe hypoglycemia, recurrent hypoglycemia, hypoglycemia unawareness, and poor postprandial glycemic control remain significant challenges.

Non-insulin anti-diabetic medications have often been considered as adjunctive to insulin therapy to improve metabolic control in T1D. ${ }^{6}$ The aim of such adjunctive therapy would be:

- to improve glycemic control;

- to minimize weight gain;

- to reduce insulin dose requirement;

- to improve postprandial glucose control; and

- to improve cardiovascular and renal risk.

Drugs which have been explored for their role in T1D, with limited success, include pramlintide, metformin, pioglitazone, glucagon-like peptide 1 (GLP-1) receptor agonists, SGLT2 inhibitors and dual SGLT1 and SGLT2 inhibitors.5,6 Pramlintide, an amylin analogue, is the only drug currently approved for use as adjunctive therapy in T1D. Trials of GLP1 receptor agonists have demonstrated small reductions in glycated haemoglobin (HbA1c) levels accompanied by weight loss and reduced insulin dose but a significantly increased risk of hypoglycemia. ${ }^{6,7}$ In the recently published 
multicentre trial with metformin in T1D adults with high cardiovascular risk (REMOVAL; NCT01483560), metformin demonstrated reductions in body weight, low density lipoprotein (LDL) cholesterol and maximal carotid intima media thickness (CIMT), though the mean cIMT (primary outcome) was not affected. ${ }^{8}$

\section{Rationale for use of Sodium-glucose Co-transporter-2 Inhibitors in Type 1 Diabetes}

Sodium-glucose co-transporter-2 (SGLT2) inhibitors reduce renal glucose reabsorption and promote glucouresis and natriuresis. In trials of type 2 diabetes (T2D) patients, they have demonstrated significant glucose-lowering efficacy and moderate reductions in blood pressure, lipids, uric acid, and body weight (predominantly loss of fat mass). These benefits have been demonstrated even when SGLT2 inhibitors have been used as add-on to insulin therapy in T2D, with no increased risk of hypoglycemia and an attenuation of insulin-related weight gain. Longterm outcome studies and real-world data have suggested that they have cardio-protective and potential reno-protective benefits in patients with high cardiovascular risk. ${ }^{9}$ Since the mechanism of action does not depend on endogenous insulin secretion and there are multiple pleiotropic benefits, SGLT2 inhibition is an attractive target in T1D management, as adjunctive to insulin. Likely benefits are better glycemic control, reduced insulin dose requirements, weight and blood pressure reduction, reduction in insulin resistance and possible cardio-protection and reno-protection.

\section{Clinical evidence with sodium-glucose co-transporter-2 inhibitors in type 1 diabetes}

In animal models of T1D, SGLT2 inhibitors have demonstrated glucoselowering effects. Empagliflozin increased urinary glucose excretion (UGE) and improved glycemic control in streptozotocin-induced T1D rats. ${ }^{10,11}$ In T1D mice, empagliflozin treatment significantly improved glucose tolerance with improvements in mRNA and serum insulin levels. ${ }^{10}$ The authors hypothesized that empagliflozin may help in the preservation of $\beta$-cell mass, evidenced by reduction in $\beta$-cell apoptosis and stimulation of $\beta$-cell proliferation, possibly due to protection of $\beta$-cells from glucotoxicity-induced oxidative stress.

Several small pilot studies of SGLT2 inhibitors and real-world case reports suggest that SGLT2 inhibitors improve glycemic control in T1D. ${ }^{12}$ Dapagliflozin increased UGE by 72-88 g per 24 hours in a dose ranging study in 70 T1D adults with reduction in daily insulin dose requirements by $16.2-19.3 \%{ }^{13,14}$ There were significant reductions in 24-hour average glucose and glycemic variability, without any unexpected short-term safety concerns over 2 weeks. ${ }^{13,14}$ In another 24-week study in T1D subjects, dapagliflozin reduced fasting glucose, postprandial glucose, HbA1C, total cholesterol, LDL cholesterol, and triglycerides with no significant adverse events. ${ }^{15}$ Similar benefits were seen when dapagliflozin was added to ongoing insulin and liraglutide therapy. ${ }^{16}$

Empagliflozin significantly lowered 24-hour UGE in 75 T1D individuals. Compared to placebo, the $\mathrm{HbA} 1 \mathrm{C}$ reduction was -0.35 to $-0.49 \%$, total daily insulin dose was reduced by -0.07 to $-0.09 \mathrm{U} / \mathrm{kg}$, and weight reduction was -1.5 to $-1.9 \mathrm{~kg}$. The rate of symptomatic hypoglycemia or hypoglycemia requiring assistance was not increased. ${ }^{17}$ The 24 -hour mean glucose area under curve was significantly reduced, compared to placebo: adjusted mean difference was $-30.2 \mathrm{mg} / \mathrm{dl} / \mathrm{h}$ ( $95 \%$ confidence interval [Cl] -42.2 to -18.2) with empagliflozin $10 \mathrm{mg}$ and $-33.0 \mathrm{mg} / \mathrm{dl} / \mathrm{h}(95 \% \mathrm{Cl}-44.8$ to -21.1$)$ with empagliflozin $25 \mathrm{mg}$. Glucose variability was significantly decreased, time spent in target glucose range was increased and there was no increase in time spent in hypoglycemia. ${ }^{18}$

In an 18-week, phase 2 study including 351 patients on multiple daily injections or CSII, canagliflozin was associated with decreases in HbA1C, insulin dose and body weight. ${ }^{19}$ Eighty-nine of these participants underwent CGM and canagliflozin use was associated with improved indices of glycemic variability. ${ }^{20}$ In another CGM study, significant reductions in mean blood glucose, CGM standard deviation, time spent in hyperglycemia, HbA1c, weight, systolic blood pressure (SBP), and total daily insulin dose were reported in 27 patients with canagliflozin as add-on to insulin. ${ }^{21}$ Similar benefits have been reported with ipragliflozin and remogliflozin in small pilot studies. ${ }^{14,22}$

In a recent meta-analysis of three randomized controlled trials including 178 patients, SGLT2 inhibitor use was associated with reduction in fasting blood glucose and insulin dosage, with no significant risk of urinary tract infections, genital infections or diabetic ketoacidosis (DKA). ${ }^{23}$ In another systematic review of all studies published till March 2017, SGLT2 inhibitors led to overall reduction of HbA1c (up to $0.49 \%$ ) and weight (up to $2.7 \mathrm{~kg}$ ) and lower total daily insulin dose, with lower incidence of hypoglycemia. ${ }^{24}$

Empagliflozin resulted in a decrease in arterial stiffness in young patients with T1D. ${ }^{25}$ In another study, empagliflozin significantly reduced renal hyperfiltration, with decline in plasma nitric oxide (NO) and effective renal plasma flow over 8 weeks. ${ }^{26}$ In a mouse model (Akita mice) of T1D, dapagliflozin resulted in significant reduction in mesangial matrix expansion and renal interstitial fibrosis, along with reduction in hyperglycemiainduced oxidative stress, compared to insulin. ${ }^{14,27}$ In T2D outcome trials, empagliflozin and canagliflozin have demonstrated cardio-protection and reno-protection. This leads to the hope that SGLT2 inhibitors may reduce nephropathy progression and cardiovascular risk in T1D as well.

However, most studies have been of short duration and small sample size, and no conclusions can be made about the long-term efficacy and safety of SGLT2 inhibitors in T1D. There are limited data that SGLT2 inhibitors improve average glucose and glycemic variability, reduce postprandial hyperglycemia, promote weight reduction, and reduce total daily insulin dose requirement without increased risk of hypoglycemia in T1D.

\section{Safety concerns-risk of diabetic ketoacidosis}

SGLT2 inhibitors are associated with an increased risk of genital mycotic infections, urinary tract infections, and volume depletion. Recent studies in T2D have raised concerns about their long-term effects on bone health and increased risk of fractures and lower extremity amputations, as seen with canagliflozin. An assessment of the impact of chronic SGLT2 inhibition on bone health and amputation risk in T1D is especially pertinent as these patients are at high risk of osteoporosis, fractures, neuropathy, peripheral vascular disease, and amputations. ${ }^{28,29}$

Most importantly, there has been growing concern about the increased risk of ketoacidosis associated with the use of SGLT2 inhibitors. In animal studies, phlorizin induced hyperketonemia in rats and decreased the renal clearance of acetoacetate in dogs. Ipragliflozin led to significant increase in free fatty acid (FFA) and $\beta$-hydroxybutyrate levels in obese mice fed on a high-fat diet. Tofogliflozin caused a dose-dependent increase in acetoacetate and $\beta$-hydroxybutyrate levels in patients with T2D. ${ }^{30}$ 
Figure 1: Potential mechanisms for sodium-glucose co-transporter-2 inhibitor associated increased risk of diabetic ketoacidosis

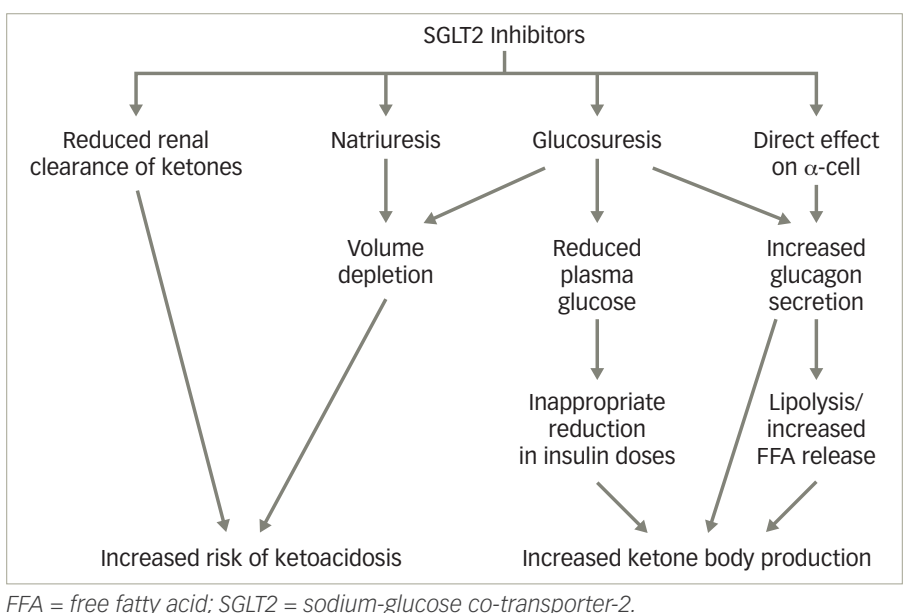

FFA $=$ free fatty acid $:$ SGLT2 = sodium-glucose co-transporter -2

In an 8-week study of empagliflozin in T1D, 5\% patients were withdrawn from the study when they developed diabetic ketoacidosis. Similarly, an increased incidence of ketoacidosis has been reported with canagliflozin (5.1\% with canagliflozin100 mg and 9.4\% with canagliflozin 300 mg versus $0 \%$ with placebo) in T1D trials. Predisposing factors included concurrent infection, insulin pump failure or missed insulin doses. ${ }^{31}$ In a phase 2 study of 2 weeks with dapagliflozin, a dose reduction in daily insulin dose of more than $20 \%$ was associated with higher $\beta$-hydroxybutyrate levels at days 7 and $14 .{ }^{32}$ In another trial, dapagliflozin was associated with significant increases in FFA, acetoacetate and $\beta$-hydroxybutyrate levels. ${ }^{16}$ Dapagliflozin increased the $\beta$-hydroxybutyrate levels to $0.6-1.0 \mathrm{mmol} / \mathrm{L}$ in 6 out of $33 \mathrm{~T} 1 \mathrm{D}$ patients during the study period of 24 hours. $^{33}$

There have been several case reports of SGLT2 inhibitor-associated DKA in individuals with both T1D and T2D. In several of these reports, hyperglycemia has not been marked, often leading to a delay in detection of DKA. This entity of euglycemic diabetic ketoacidosis has previously been reported with decreased carbohydrate intake, alcohol consumption, and during pregnancy. In May 2015, the US Food and Drug Administration (FDA) issued a warning about the increased risk of diabetic ketoacidosis associated with SGLT2 inhibitors. In a recent review of FDA adverse event reporting data, the authors found 46,144 , and 450 reports of ketoacidosis associated with empagliflozin, dapagliflozin, and canagliflozin. ${ }^{34}$ There have been some case reports from southeast Asian countries ${ }^{35,36}$ and reports of ketonemia and ketonuria in patients from India. ${ }^{37,38}$

\section{Dual sodium-glucose co-transporter-1 and sodium-glucose co-transporter-2 inhibitors}

Sotagliflozin is a dual SGLT1 and SGLT2 inhibitor, under development for use as adjunctive therapy in T1D. ${ }^{39}$ SGLT1 mediates the intestinal absorption of glucose and galactose and renal glucose reabsorption distal to SGLT2 effect. Thus, sotagliflozin reduces renal glucose reabsorption via SGLT2 inhibition and glucose absorption from the gastrointestinal tract via SGLT1 inhibition. Inhibition of SGLT1 thus reduces postprandial glucose excursions and also stimulates the release of glucagon-like peptide 1 (GLP1) and polypeptide YY (PYY), which offers further therapeutic benefits. ${ }^{40}$
Table 1: Minimizing the risk of diabetic ketoacidosis associated with the use of sodium-glucose co-transporter-2 inhibitors in type 1 diabetes

\begin{tabular}{l|l|}
\hline Physician Education & Patient Education \\
\hline Not approved, use is off-label. Discuss & Avoid skipping insulin doses. \\
the risks with the patient. & Ensure good hydration. \\
Avoid significant (>20\%) reductions in & Ensure adequate carbohydrate intake. \\
insulin doses. & Follow sick day guidelines. \\
Discontinue SGLT2 inhibitors 3 days prior & Do not reduce or skip insulin doses in \\
to elective surgery. & any acute illness, contact the healthcare \\
Discontinue SGLT2 inhibitors in case & practitioner as soon as possible. \\
of acute illness, trauma, gastroparesis, & Regular urine ketone monitoring, \\
osmotic symptoms, significant weight & especially during any illness, or if \\
loss or reduced carbohydrate intake. & the patient has nausea, vomiting or \\
Ketoacidosis may present even & abdominal discomfort. \\
with lower plasma glucose values- & Urine ketone should be tested even if \\
"euglycemic DKA". Keep a high index of & blood glucose is not very high. \\
suspicion in such patients. & \\
Patients on SGLT2 inhibitors will & \\
require dextrose infusion, to ensure & \\
that adequate insulin is administered & \\
to prevent DKA or recover from & \\
ketoacidosis. & \\
\hline
\end{tabular}

$D K A=$ diabetic ketoacidosis; $S G L T 2$ = sodium-g/ucose co-transporter-2.

In small pilot studies over 4-12 weeks, sotagliflozin improved glycemic control with significant improvements in pre- and post-meal glucose levels without increase in hypoglycemic events, documented by CGM. The reduction in post-meal glucose was associated with reduction in bolus insulin doses, not seen earlier with empagliflozin or dapagliflozin. 39,41 DKA events were higher with sotagliflozin. In a recent international phase 3 double blind randomized trials in patients with T1D using multiple subcutaneous insulin or pump therapy, the in Tandem 2 study (782 participants), the efficacy and safety of sotagliflozin $400 \mathrm{mg}$, sotagliflozin $200 \mathrm{mg}$ versus placebo was assessed over 52 weeks. There was statistically significant lowering of HbA1C $(-0.49 \%$ with sotagliflozin $400 \mathrm{mg},-0.43 \%$ with sotagliflozin $200 \mathrm{mg}$ and $-0.08 \%$ with placebo) with increased risk of genital mycotic infections and diarrhea events. The incidence of DKA was higher with sotagliflozin (3.1\% with sotagliflozin $400 \mathrm{mg}, 1.1 \%$ with sotagliflozin $200 \mathrm{mg}$ and $0 \%$ with placebo). ${ }^{42}$

\section{Mechanism for diabetic ketoacidosis}

SGLT2 inhibitors may increase the risk of DKA in T1D both directly and indirectly. ${ }^{30,43}$ SGLT2 inhibitor use may lead to deceptively lower blood glucose values and prompt inappropriate reduction in insulin doses to mitigate the risk of hypoglycemia. Resultant insulinopenia increases lipolysis, FFA release and ketogenesis. SGLT2 inhibitors lower the renal threshold for glucose to $70 \mathrm{mg} / \mathrm{dl}$, causing large amounts of glucose to be lost in urine. Therefore, blood glucose values may not be significantly elevated in patients with DKA, with the potential risk of a delay in diagnosis.

SGLT2 inhibitors may decrease the renal clearance of ketone bodies with increase in plasma ketone levels. Phlorizin was shown to promote renal tubular reabsorption of acetoacetate in dogs, but the clearance of $\beta$-hydroxybutyrate was not measured. ${ }^{44}$ If SGLT2 inhibitors delay renal clearance of ketones, this may further delay the diagnosis of DKA. 
Glucouresis may lead to compensatory rise in plasma glucagon levels, resulting in increased gluconeogenesis and FFA release. SGLT2 is expressed in pancreatic $\alpha$-cells, and SGLT2 inhibitors increase glucagon secretion directly through ATP-sensitive potassium (KATP) channel activation. ${ }^{45}$ The resultant increase in glucagon/insulin ratio further promotes ketogenesis. ${ }^{30}$ Figure 1 delineates the potential mechanisms by which SGLT2 inhibitors may increase the risk of ketoacidosis in patients with T1D.

In most cases reported so far there were clear precipitating factors which could have triggered the ketoacidotic state. The risk factors described include: T1D/latent autoimmune diabetes or significant insulinopenia in T2D, insulin omission, or significant dose reduction, severe acute illness, surgery, trauma, alcohol intake, extensive exercise, dehydration, or low carbohydrate diet. ${ }^{46-48}$

\section{How to minimize the risk of diabetic ketoacidosis with sodium-glucose co-transporter-2 inhibitors}

The off-label use of SGLT2 inhibitors in T1D is increasing, despite the lack of adequate safety data, and this is a cause for concern. There is no experience with the use of SGLT2 inhibitors in the pediatric population and it should not be used in such settings at all. Any use of SGLT2 inhibitors in T1D, at present, is off-label and the risk of diabetic ketoacidosis along with the lack of long-term safety data must be discussed at length with the patient. ${ }^{49}$ If their use is being considered at all, the practitioner should be very cautious and ensure appropriate patient education. There is also a need for all healthcare practitioners to keep a high index of suspicion for DKA in any patient using SGLT2 inhibitors and presenting with an acute medical or surgical illness. This is true even for those patients in whom blood glucose is apparently lower than one would expect in DKA, since euglycemic DKA has been frequently reported in such patients. In Table 1, we recommend strategies to minimize the risk of DKA associated with the use of SGLT2 inhibitors. These strategies will be useful in T2D patients as well.

\section{Summary}

While SGLT2 inhibitors have the potential to improve glycemic control, reduce insulin dose requirements and reduce insulin-associated weight gain, the risk of DKA must be considered and adequate measures taken to prevent its occurrence. Any use of SGLT2 inhibitors in T1D is off-label and the risks must be discussed with the patient. $\square$
1. Libman IM, Pietropaolo M, Arslanian SA, et al., Changing prevalence of overweight children and adolescents at onset of insulin-treated diabetes, Diabetes Care, 2003:26:2871-5

2. Liu LL, Lawrence JM, Davis C, et al., Prevalence of overweight and obesity in youth with diabetes in USA: the SEARCH for Diabetes in Youth study, Pediatr Diabetes, 2010;11:4-11

3. Purnell JQ, Zinman B, Brunzell JD, et al., The effect of excess weigh gain with intensive diabetes mellitus treatment on cardiovascular disease risk factors and atherosclerosis in type 1 diabetes mellitus: results from the Diabetes Control and Complications Trial/ Epidemiology of Diabetes Interventions and Complications Study (DCCT/EDIC) study Circulation, 2013:127:180-7.

4. Wajchenberg BL, Feitosa AC, Rassi N, et al., Glycemia and cardiovascular disease in type 1 diabetes mellitus, Endocr Pract, 2008;14:912-23.

5. Bode BW, Garg SK, The emerging role of adjunctive noninsulin antihyperglycemic therapy in the management of type 1 diabetes, Endocr Pract, 2016;22:220-30.

6. Comee $\mathrm{M}$, Peters $\mathrm{A}$, The changing therapeutic armamentarium for patients with type 1 diabetes, Curr Opin Endocrinol Diabetes Obes, 2016:23:106-10.

7. Dejgaard TF, Frandsen CS, Holst JJ, Madsbad S, Liraglutide for type 1 diabetes, Expert Opin Biol Ther, 2016;16:579-90.

8. Petrie JR, Chaturvedi N, Ford I, et al., Cardiovascular and metabolic effects of metformin in patients with type 1 diabetes (REMOVAL): a double-blind, randomised, placebo-controlled trial Lancet Diabetes Endocrinol, 2017:5:597-609.

9. Minze MG, Will K, Terrell BT, et al. Benefits of SGLT2 Inhibitors beyond glycemic control - A focus on metabolic, cardiovascular, and renal outcomes, Curr Diabetes Rev, 2017. doi: 10.2174/15733 99813666170816142351

10. Cheng ST, Chen L, Li SY, et al., The effects of empagliflozin, an SGLT2 inhibitor, on pancreatic $\beta$-cell mass and glucose homeostasis in type 1 diabetes, PLOS One, 2016;11:0147391.

11. Luippold G, Klein T, Mark M, Grempler R, Empagliflozin, a nove potent and selective SGLT-2 inhibitor, improves glcaemic control alone and in combination with insulin in streptozotocin-induced diabetes rats, a model of type 1 diabetes mellitus, Diabetes Obes Metab, 2012:14:601-7.

12. Bell DS, Case reports that illustrate the efficacy of SGLT2 inhibitors in the type 1 diabetic patient, Case Rep Endocrinol, 2015:676191.

13. Henry RR, Rosenstock J, Edelman S, et al., Exploring the potential of the SGLT2 inhibitor dapagliflozin in type 1 diabetes: a randomized, double-blind, placebo-controlled pilot study, Diabetes Care, 2015:38:412-9.

14. Pafili K, Maltezos E, Papanas N, Dapagliflozin for the treatment of type 1 diabetes mellitus, Expert Opin Investig Drugs, 2017:26:873-81.

15. Tamez HE, Tamez AL, Garza LA, et al., Dapagliflozin as an adjunct therapy to insulin in the treatment of patients with type 1 diabetes mellitus, J Diabetes Metab Disord, 2015;14:78.

16. Kuhadiya ND, Ghanim $H$, Mehta A et al., Dapagliflozin as additional treatment to liraglutide and insulin in patients with type 1 diabetes, I Clin Endocrinol Metab, 2016:101:3506-15.

17. Pieber TR, Famulla $S$, Eilbracht J, et al., Empagliflozin as adjunct to insulin in patients with type 1 diabetes: a 4-week, randomized, placebo-controlled trial (EASE-1), Diabetes Obes Metab, 2015;17:928-35.
18. Famulla S, Pieber TR, Eilbracht J, et al., Glucose Exposure and Variability with Empagliflozin as Adjunct to Insulin in Patients with Type 1 Diabetes: Continuous Glucose Monitoring Data from a 4-Week, Randomized, Placebo-Controlled Trial (EASE-1), Diabetes Technol Ther, 2017:19:49-60.

19. Henry RR, Thakkar P, Tong C, et al., Efficacy and safety of canagliflozin, a sodium-glucose contransporter 2 inhibition, as add-on to insulin in patients with type 1 diabetes, Diabetes Care, 2015;38:2258-65.

20. Rodbard HW, Peters AL, Slee A, et al. The effect of canagliflozin, a sodium glucose transporter 2 inhibitor, on glycemic end points assessed by continuous glucose monitoring of patient-reported out 1 diabetes, Diabetes Care, 2017:40:11-180

21. Argento NB, Nakumura K, Glycemic effects of SGLT-2 inhibitor canagliflozin in type 1 diabetes patients using the DEXCOM G4 platinum CGM, Endocr Pract, 2016;22:315-22.

22. Tahara A, Kurosaki E, Yokono M, et al., Effects of sodium-glucose cotransporter 2 selective inhibitor ipragliflozin on hyperglycemia oxidative stress, inflammation and liver injury in streptozotocininduced type 1 iabetes rats, I Pharm Pharmacol, 2014:66:975-87.

23. Yingying $Y$, Hui $P, B O W$, et al., Efficacy and safety of SGLT2 inhibitors in patients with type 1 diabetes: a meta-analysis of randomized controlled trials, Chin Med Sci J, 2017;32:22-27.

24. Ahmed-Sarwar N, Nagel AK, Leistman S, Heacock K, SGLT-2 inhibitors: Is there a role in type 1 diabetes mellitus management? Ann Pharmacother, 2017:51:791-6.

25. Cherney DZ, Perkins BA, Soleymanlou N, et al., The effect of empagliflozin on arterial stiffness and heart rate variability in subjects with uncomplicated type 1 diabetes mellitus, Cardiovas Diabetol, 2014:13:28.

26. Cherney DZ, Perkins BA, Soleymanlou N, et al., Rena hemodynamic effect of sodium-glucose cotransporter 2 inhibition in patients with type 1 diabetes mellitus, Circulation, 2014:129:587-97.

27. Hatanaka T, Ogawa D, Tachibana H, et al., Inhibition of SGLT2 alleviates diabetic nephropathy by suppressing high glucoseinduced oxidative stress in type 1 diabetic mice, Pharmacol Res Perspect, 2016:4:e00239.

28. Weber DR, Schwartz G, Epidemiology of skeletal health in type 1 diabetes, Curr Osteoporos Rep, 2016;14:327-36.

29. Melendez-Ramirez LY, Richards RJ, Cefalu WT, Complications of type 1 diabetes, Endocrinol Metab Clin North Am, 2010;39:625-40.

30. Singh AK, Sodium-glucose co-transporter-2 inhibitors and euglycemic ketoacidosis: Wisdom of hindsight, Indian J Endocrinol Metab, 2015:19:722-30.

31. Peters AL, Henry RR, Thakkar $P$, et al., Diabetic ketoacidosis with canagliflozin, a sodium glucose cotransporter 2 inhibitor, in patients with type 1 diabetes, Diabetes Care, 2016;39:532-8

32. Henry RR, Dandona P, Pettus J, et al., Dapagliflozin in patients with type 1 diabetes: a post-hoc analysis of the effect of insulin dose adjustments on 24-hour continuously monitored mean glucose and fasting beta-hydroxybutyrate levels from a phase lla pilot and

33. Biester T, Asxhemeier B, Fath M, et al., Effects of dapagliflozin on insulin requirement, glucose excretion and $\beta$-hydroxybutyrate levels are not related to baseline $\mathrm{HbA1C}$ in youth with type 1 diabetes, Diabetes Obes Metab, 2017;19:1635-9.
34. D'Elia JA, Segal AR, Bayliss GP, Weinrauch LA, Sodium-glucose cotransporter-2 inhibition and acidosis in patients with type 2 diabetes: a review of US FDA data and possible conclusions, Int $\rfloor$ Nephrol Renovasc Dis, 2017:10:153-8.

35. Bader N, Mirza L, Euglycemic diabetic ketoacidosis in a 27 year-old female patient with type 1 diabetes treated with sodiumglucose cotransporter-2 (SGLT2) inhibitor Canagliflozin, Pak J Med SCi, 2016;32:786-8.

36. Tahir $\mathrm{H}$, Wani A, Daruwalla V, et al., Euglycemic diabetic ketoacidosis and severe acute kidney injury secondary to off label use of sodium glucose cotransporter-2 inhibitor in a type-1 diabetic patient, J Avub Med Coll Abbottabad, 2015:27:923-4.

37. Ghosh A, Gupta R, Misra A, Ketonuria/ ketonemia associated with the use of sodium-glucose cotransporter 2 (SGLT-2) inhibitors in type 2 diabetes: a report of three cases from New Delhi, India, J Diabetes, 2016;8:738-9.

38. Kelwade J, Sethi BK, Nagesh SV, Vaseem A, A case of "pseudoketoacidosis", Indian J Endocrinol Metab, 2014:18:743.

39. Sands AT, Zambrowicz BP, Rosenstock J, et al., Sotagliflozin, a dual SGLT1 and SGLT2 inhibitor; as adjunct therapy to insulin in type 1 diabetes, Diabetes Care, 2015;38:1181-8.

40. Cariou B, Charbonnel B, Sotagliflozin as a potential treatment for type 2 diabetes mellitus, Expert Opin Investig Drugs, 2015;24:1647-56.

41. Bode BW, Banks P, Sawhney S, Strumph P, the Sotagliflozin JDRF Study Writing group. Sotagliflozin (SOTA) is a dual SGLT1 and SGIT2 inhibitor in phase 3 development. American Diabetes Association, 77th Sessions, San Diego. Late Breaking Abstracts Association, 77th Sessions,

42. Danne T, Cariou B, Banks P, Sawhney S, Strumph P, the Sotagliflozin in Tandem2 writing group. 24-week efficacy and safety of sotagliflozin, a dual SGLT1 and SGLT2 inhibitor, as adjunct therapy to insulin in type 1 diabetes (in Tandem2). American Diabetes Association, 77th Sessions, San Diego. Late Breaking Abstracts June 2017, 66(1A): 146-LB.

43. Taylor SI, Blau JE, Rother KI, SGLT2 inhibitors may predispose to ketoacidosis, J Clin Endocrinol Metab, 2015:100:2849-52.

44. Cohen JJ, Berglund F, Lotspeich WD, Renal tubular reabsorption of acetoacetate, inorganic sulfate and inorganic phosphate in the dog as affected by glucose and phlorizin, Am J Physiol 1956;184:91-6.

45. Kibbey RG, SGLT-2 inhibition and glucagon: Cause for alarm? Trends Endocrinol Metab, 2015:26:337-8.

46. Goldenberg RM, Berard LD, Cheng AY, et al., SGLT2 inhibitorassociated diabetic ketoacidosis: clinical review and recommendations for prevention and diagnosis, Clin Ther 2016;38:2654-64.e1

47. Burke KR, Schumacher CA, Harpe SE, SGLT2 inhibitors: systematic review of diabetic ketoacidosis and related risk factors in the primary literature, Pharmacotherapy, 2017:37:187-94.

48. Kelmenson DA Burr K Azhar Y et al, Euglycemic diabetic ketoacidosis with prolonged glucosuria associated with the sodium-glucose cotransporter-2 canagliflozin, J Investig Med High Impact Case Rep, 2017:5:2324709617712736.

49. Kalra S, Sahay R, Gupta R, Sodium glucose transporter 2 (SGLT2) inhibition and ketogenesis, Indian J Endocrinol Metab 2015;19:524-8 